\title{
Circuit
}

Musiques contemporaines

\section{Störungen (2011) de David Hudry, ou la confrontation des diapasons à travers l'emploi jumelé d'instruments baroques et modernes}

\section{Störungen (2011) by David Hudry, or, The Confrontation of Tunings through the Combined Use of Baroque and Modern Instruments.}

\section{François-Xavier Féron}

Volume 28, numéro 2, 2018

Instrumentarium baroque : précédence et créativité

URI : https://id.erudit.org/iderudit/1051292ar

DOI : https://doi.org/10.7202/1051292ar

Aller au sommaire du numéro

Éditeur(s)

Circuit, musiques contemporaines

ISSN

1183-1693 (imprimé)

1488-9692 (numérique)

Découvrir la revue

Citer cet article

Féron, F.-X. (2018). Störungen (2011) de David Hudry, ou la confrontation des diapasons à travers l'emploi jumelé d'instruments baroques et modernes. Circuit, 28(2), 53-67. https://doi.org/10.7202/1051292ar
Résumé de l'article

Dans Störungen (2011), le compositeur français David Hudry explore un nouveau genre de mixité instrumentale en réunissant deux instrumentistes baroques et six instrumentistes modernes. Composée dans le cadre de la huitième édition de l'Ensemble Academy Freiburg organisée conjointement par l'ensemble recherche et le Freiburger Barockorchester, cette oeuvre explore l'idée de dysfonctionnement et de dédoublement en confrontant deux types d'instrumentarium, l'un baroque accordé à $415 \mathrm{~Hz}$ et l'autre moderne accordé à $440 \mathrm{~Hz}$. Cet article, qui s'articule autour d'un long entretien que le compositeur nous a accordé au début de l'année 2018, revient sur la genèse de l'oeuvre et souligne les enjeux et défis compositionnels propres à ce type d'effectif instrumental. 


\section{Störungen (2011) de David Hudry, ou la confrontation des diapasons à travers l'emploi jumelé d'instruments baroques et modernes}

François-Xavier Féron

Though it is not programmatic, the music of David Hudry mostly springs from some informal background narrative. In Störungen (2011) it is the confrontation between six modern and two Baroque instruments (tuned to $415 \mathrm{~Hz}$ ), which one could be tempted to view as the ripieno and concertino respectively, were it not for the composer's intentional reassignment of roles. This provides both the scenario and a clear formal envelope: a «schizophrenic confrontation of materials» glides towards an «attempt at fusion ${ }^{1}$.

\section{Prologue : quelques mots au sujet de David Hudry}

En 2016, David Hudry² a reçu le prix «jeune compositeur» de la Ernst von Siemens Music Foundation, grâce auquel il put graver son premier disque monographique publié par l'étiquette allemande col legno3. Interprétées par différents ensembles européens de renom (Ensemble Modern, ensemble recherche, Orchestre philharmonique de Radio France, Ensemble intercontemporain), les quatre œuvres figurant sur cet opus discographique permettent d'apprécier quelques-unes des caractéristiques essentielles du langage musical de David Hudry: recherches harmoniques et mélodiques, mise en scène de différents matériaux et gestes musicaux pour former une trame narrative, jeux d'opposition et de contraste, attachement à la pulsation, virtuosité rythmique...

L'écriture de David Hudry se focalise sur une dramaturgie musicale élaborée à partir de personnages et objets musicaux hétérogènes. Inspirée par les arts graphiques, et notamment par les réflexions de P. Klee et W. Kandinsky, sa musique
1. Rigaudière, 2017, p. 46.

2. Né en 1978, le compositeur français David Hudry mène, parallèlement à sa formation au Conservatoire de Montpellier, des études de musicologie à l'Université Paul Valéry dans cette même ville. En 2002, il obtient son Agrégation de Musique et entre au CSNMD de Paris où il intègre d'abord la classe d'analyse supérieure puis les classes de composition - nouvelles technologies (L. Naon, T. Mays et Y. Geslin) et de composition (E. Nunes et S. Gervasoni). En 2006, il suit le cursus de l'Ircam (Y. Maresz, M. Malt, J. Lochard et E. Jourdan) où il explore différents outils d'aide à la composition et élabore une réflexion sur les enjeux esthétiques liés aux nouvelles technologies. Particulièrement sensible au rapport entre l'écriture instrumentale, son déploiement et son prolongement dans l'électronique, David Hudry recherche très tôt une forme d'interaction vivante entre l'interprète et la machine, et en fait l'un des axes de son travail de composition. http://davidhudry.com/ bio/ (consulté le 3 avril 2018).

3. Le disque Durchgang (col legno WWE 1 CD 40418, 2017) est accompagné 
d'un remarquable livret bilingue allemand-anglais dans lequel sont reproduites de nombreuses esquisses du compositeur. https:// www.col-legno.com/en/shop/40418david-hudry-durchgang (consulté le 3 avril 2018).

4. http://davidhudry.com/bio/ (consulté le 3 avril 2018).

5. Notre entretien (Féron, 2018a) a duré un peu plus de deux heures et demie. Une fois intégralement retranscrit, nous avons sélectionné et agencé plusieurs extraits suivant différentes thématiques qui nous ont permis d'établir le squelette de ce texte. Une première version a alors été envoyée au compositeur pour qu'il puisse reformuler ses propos s'il en ressentait le besoin. Toutes les citations mises entre guillemets et insérées en italiques dans le corps du texte proviennent exclusivement de notre entretien avec le compositeur. Un supplément web (https://www. revuecircuit.ca/web/) vient compléter la lecture de cet article afin que les lecteurs puissent aussi écouter des extraits sonores de Störungen tout en consultant la partition.

6. https://www.ensemble-recherche. de/lernen/ensemble-akademie/ s'articule autour de figures et de gestes qui génèrent des tensions et donnent un caractère visuel à la matière sonore. Fasciné par tout ce que la couleur apporte comme relief et expression aux gestes musicaux, David Hudry attache une importance significative à l'harmonie et à sa capacité à agir directement sur les sens et l'émotion. À la fois rigoureuse et empreinte d'un lyrisme totalement assumé, sa musique se fait l'écho de sa personnalité et met en scène une expression intérieure privilégiant les jeux d'oppositions et de ruptures ${ }^{4}$.

Störungen (2011), la seconde pièce du disque, met en scène des jeux d'oppositions et de ruptures, non seulement en ce qui a trait aux matériaux sonores, mais aussi à travers l'effectif instrumental convoqué par le compositeur. Créée le 7 septembre 2011 dans le cadre de la huitième édition de l'Ensemble Academy Freiburg organisée conjointement par l'ensemble recherche et le Freiburger Barockorchester, cette œuvre réunit instruments baroques accordés à $415 \mathrm{~Hz}$ et instruments modernes accordés à $440 \mathrm{~Hz}$. Cette forme de mixité instrumentale n'est pas inédite mais reste inusuelle. Pourtant elle ouvre de nouvelles perspectives compositionnelles, tant sur le plan de la structuration harmonique ou mélodique avec la présence de deux diapasons que sur la couleur sonore grâce à l'association d'instruments anciens et modernes. Comment ce projet est-il né? De quelles manières le compositeur a-t-il abordé cette mixité instrumentale? Quels avantages et quelles difficultés impliquent un tel instrumentarium? Pour répondre à ces interrogations, nous sommes allé à la rencontre du compositeur qui nous a reçu à son domicile, dans la région parisienne, le 5 janvier $2018^{5}$.

\section{L'Ensemble Academy Freiburg}

C'est à Freiburg-im-Breisgau, ville allemande située au pied des montagnes de la Forêt-Noire, que résident le Freiburger Barockorchester, réputé pour ses interprétations historiquement informées des répertoires baroques et classiques, et l'ensemble recherche, tourné vers la musique de notre temps. Depuis 2004, ces deux formations de renommée internationale proposent chaque année, dans le cadre de l'Ensemble Academy Freiburg, des classes de maître et des ateliers révélant les spécificités de leurs répertoires respectifs, mais aussi les connexions qui peuvent exister dans leur pratique musicale. Cette collaboration unique sur la scène internationale s'est renforcée en 2012 avec la construction de l'Ensemblehaus, un lieu partagé par les deux formations où se tient dorénavant leur académie d'été :

Far more than two musical directions juxtapositioned [sic], what matters in the Academy is a mutual interfusion of old and new, and the mutual artistic inspiration of differing performance practices - leading to the premiering of works for Baroque and modern instruments ${ }^{6}$. 
En 2010, l'académie lance un concours de composition «Concertare», précisant dans l'appel à candidature que les œuvres devront obligatoirement mêler instruments anciens accordés à $415 \mathrm{~Hz}$ et instruments modernes accordés à $440 \mathrm{~Hz}$. Plus de soixante projets ont été évalués par le jury composé de Rebecca Saunders, Jörg Widmann, Andreas Staier, Markus Hinterhäuser, Martin Fahlenbock et Karl Kaiser. Les trois projets retenus furent ceux déposés par Mark Barden, Aaron Einbond et David Hudry; les trois compositeurs reçurent alors une commande conjointe de l'ensemble recherche et du Freiburger Barockorchester (Figure 1).

FIGURE 1 OEuvres commandées par l'ensemble recherche et le Freiburger Barockorchester et créées en septembre 2011 dans le cadre de la huitième édition de l'Ensemble Academy Freiburg ${ }^{8}$.

\begin{tabular}{|l|l|l|}
\hline & \multicolumn{1}{|c|}{$\begin{array}{c}\text { Instrumentarium } \\
\text { baroque accordé à } \mathbf{4 1 5} \mathbf{~ H z}\end{array}$} & $\begin{array}{l}\text { Instrumentarium } \\
\text { moderne accordé à } \mathbf{4 4 0} \mathbf{~ H z}\end{array}$ \\
\hline $\begin{array}{l}\text { Mark Barden, } \\
\text { Tenebrae }\end{array}$ & cor, alto, contrebasse, orgue & $\begin{array}{l}\text { clarinettes, violon, violoncelle, } \\
\text { percussions }\end{array}$ \\
\hline $\begin{array}{l}\text { Aaron Einbond, } \\
\text { Paläontologie }\end{array}$ & $\begin{array}{l}\text { traverso, clavecin, viole de } \\
\text { gambe, luth }\end{array}$ & $\begin{array}{l}\text { clarinette basse, violon, } \\
\text { percussions, piano }\end{array}$ \\
\hline $\begin{array}{l}\text { David Hudry, } \\
\text { Störungen }\end{array}$ & traverso, alto & $\begin{array}{l}\text { clarinettes, violon, violoncelle, } \\
\text { percussions, piano }\end{array}$ \\
\hline
\end{tabular}

Dans le projet qu'il déposa à l'automne 2010, David Hudry spécifiait déjà l'instrumentarium de sa pièce et décrivait la manière dont il souhaitait explorer la dimension concertante entre les univers baroque et moderne qui était au cœur de ce concours de composition. "La contrainte, c'était de réunir les deux mondes - baroque et moderne-dans un projet qui pouvait se décliner de différentes façons. On pouvait mélanger des instruments baroques au sein de la formation moderne. Il n'y avait pas nécessité de les séparer, mais moi j'ai voulu le faire. L'idée du projet, c'était "Concertare». Il y avait l'idée d'un concerto grosso, ou plutôt de quelque chose qui s'en approchait. Je voulais un soliste ou deux instruments qui pourraient être dédoublés dans la formation car cette idée correspond à ma manière de composer. On peut considérer que les solistes jouent le rôle de concertino, et l'ensemble complet, le rôle du ripieno dans la tradition du concerto grosso baroque. Libre à chacun d'interpréter cela comme il l'entend... »

David Hudry a réalisé quelques esquisses en 2010 mais la phase intense de composition s'est étalée essentiellement entre janvier et avril 2011. Quelles connaissances avait-il alors des instruments anciens et comment a-t-il abordé la question des deux tempéraments?
7. Féron, 2018b.

8. Ibid. 
9. Se référer dans ce numéro à l'article de Feneyrou.

10. Le compositeur suisse Klaus Huber (1924-2017) a plusieurs fois fait appel à des instruments anciens, en particulier à partir des années 1990. La viole d'amour - souvent accordée en tiers de ton - est présente dans les différentes versions de Plainte - Die umgepflügte Zeit (1990) écrites à la mémoire de Luigi Nono mais aussi dans L'âge de notre ombre (1998), L'ombre de notre âge (1998-1999), Vida y muerte no son mundos contrarios (2007)... Dans Agnus Dei cum Recordatione (19901991), Huber fait appel à un luth de la Renaissance et à deux vièles à archet alors que dans A Voice from Guernica. . (2003), il recourt à la mandoline. David Hudry a rencontré à plusieurs reprises le compositeur suisse et a d'ailleurs consacré son prix d'analyse du CNSMD de Paris à l'une de ses œuvres: ... Inwendig voller Figur... (1970-1971) pour chœur, haut-parleurs, grand orchestre et bande magnétique.

11. Intersections (2014), écrite pour le même effectif, a été créée à Caen en mars 2014 par l'ensemble Multilatérale dans le cadre du festival "Aspects des musiques d'aujourd'hui ". Si Störungen et Intersections devaient au départ se répondre d'une certaine façon, ne serait-ce qu'à travers la similarité de l'effectif instrumental, les deux œuvres prirent finalement des directions

\section{L'ombre de Klaus Huber}

Réunir instruments baroques et modernes au sein d'une même œuvre demeure une pratique peu usitée, voire exceptionnelle, car elle n'est pas sans poser quelques défis aux compositeurs qui doivent alors travailler avec des instruments accordés suivant des diapasons différents et assimiler les spécificités techniques et sonores des deux types d'instrumentarium. David Hudry était à l'époque parfaitement novice en la matière puisqu'il n'avait jamais fait appel, avant Störungen, à des instruments anciens. Il avait en revanche entendu quelques pièces contemporaines mêlant les deux instrumentariums, notamment celles de Brice Pauset ${ }^{9}$. Mais c'est davantage avec Klaus Huber qu'il se familiarisa avec cette forme de mixité instrumentale ${ }^{10}$. "Je sais que Klaus Huber a souvent fait ce genre d'expérience avec instruments modernes et baroques. Il l'a souvent pratiqué dans sa musique. Lui, il a écrit énormément de projets dans lesquels les instruments baroques rencontrent les instruments modernes. [...] C'est quelqu'un dont j'ai toujours apprécié la poésie musicale et la manière de composer. Il avait cette fascination pour le mélange des univers. »

Dans le cadre du concours de l'Ensemble Academy, la différence entre les deux diapasons correspond exactement à un demi-ton tempéré. Cette contrainte ne génère donc pas en soi une musique microtonale - à moins de recourir à des micro-intervalles - de laquelle se dégagerait une couleur sonore bien particulière comme, par exemple, dans Ramifications (1968-1969) de György Ligeti pour douze cordes solistes; dans cette œuvre, le compositeur hongrois scinde l'effectif en deux groupes accordés respectivement à $440 \mathrm{~Hz}$ et $453 \mathrm{~Hz}$ de sorte à travailler entre autres sur les phénomènes de battement et de brouillage que permet ce léger désaccordage. Le propos de David Hudry est tout autre dans Störungen puisque la dualité des diapasons l'incita plutôt à explorer la forme du concerto grosso et la question du dédoublement: «je me suis dit simplement que j'allais travailler sur l'idée du double, en fait sur l'idée du dédoublement, qui est une idée qui m’intéresse aussi de manière générale».

\section{Störungen ou l'esquisse d'un concerto grosso pour traverso et alto baroque}

Comme nous l'avons précédemment mentionné, l'effectif instrumental de Störungen était défini dès 2010 dans le projet soumis à l'Ensemble Academy. Le choix des six instruments modernes (flûtes, clarinettes, percussions, piano, violon et violoncelle) était bien évidemment dicté par l'effectif instrumental de l'ensemble recherche mais faisait aussi écho à un autre projet compositionnel que le compositeur esquissait à la même époque ${ }^{11}$. Quant au choix des deux instruments anciens, le traverso et l'alto baroque, il s'inscrivait 
dans l'idée de construire une sorte de concerto grosso pour deux instruments appartenant à des familles distinctes mais ayant chacun leur double au sein de l'instrumentarium moderne. "Déjà je voulais deux instruments de familles différentes. C'était la première contrainte que je m'étais imposée. Et je voulais absolument qu'il y ait l'alto car c'est un instrument que j'affectionne particulièrement. J'avais déjà écrit une pièce dans laquelle je m'étais vraiment approprié l'instrument. Du coup, c'était quelque chose que je voulais poursuivre pour donner une seconde vie à des matériaux déjà travaillés avec l'alto et les recontextualiser en les confrontant à d'autres instruments, ici l'alto baroque et le violoncelle moderne. [...] Je voulais que les deux instruments solistes, dans ce qui était proposé, puissent être repris ou doublés, puissent faire l'objet de jeux de dialogue ou de confrontation avec un des instruments solistes de l'ensemble recherche.»

Si Störungen esquisse ainsi les traits d'un concerto grosso pour traverso et alto baroque, il est en revanche simplement stipulé sur la partition que l'œuvre fait appel à «un ensemble mixte d'instruments baroques et modernes». Le compositeur n’a pas souhaité mettre en avant le rôle soliste des deux instruments baroques dans l'intitulé car la forme de l'œuvre est en réalité plus ambiguë. "Ie l'ai pensée sous différents angles. Il y a des moments où l'idée du concerto ressort et des moments où je ne souhaite pas forcément qu'on sache s'il s'agit d'un concerto. Quand je compose, j'aime bien que ma musique garde sa capacité à évoluer, à se décliner sous différentes formes. [...]. Le propos du "Concertare», de l'échange solistes-ensemble, est clair dès le départ mais en même temps, il y a des moments où les matières sont tellement foisonnantes que du coup, on ne sait plus qui joue quel rôle. On n'identifie plus une relation claire entre les instruments qui participent à une activité, une énergie globale. »

\section{Structuration narrative de l'œuvre}

Störungen, d'une durée légèrement inférieure à vingt minutes, comprend sept sections (repères A à G sur la partition) qui ont des «caractères bien différents». Störungen est un mot allemand signifiant: «dysfonctionnement», «perturbation ». Si le titre a été choisi une fois l'œuvre achevée, cette idée était bien présente dans l'esprit du compositeur dès le début du processus de création. David Hudry souhaitait, en effet, mettre en scène une forme de lutte sonore entre instruments anciens et modernes, les uns perturbant les autres dans l'esprit d'un dysfonctionnement instrumental. Mais cette idée de dysfonctionnement s'exprime aussi à travers l'alternance, parfois brusque, des matériaux sonores. "La pièce commence comme cela, avec une matière agitée, foisonnante, puis tout d'un coup je fige les choses. Ensuite, j'alterne les deux 
12. Les musiciens de l'ensemble recherche occupent le fond du plateau (Christian Dierstein aux percussions, Klaus SteffesHolländer au piano, Martin Fahlenbock aux flûtes, Shizuyo Oka aux clarinettes, Melise Mellinger au violon, Åsa Åkerberg au violoncelle) alors que les deux musiciens baroques (Karl Kaiser au traverso et Barbara Maurer à l'alto baroque) sont placés devant. situations en les juxtaposant de manière abrupte. Il y a tout un jeu d'aller-retour entre ces deux états qui fait que, petit à petit, la matière agitée laisse place à de longues plages glacées. »

La dimension narrative et visuelle de Störungen repose en partie sur la confrontation conjointe des matériaux et des instrumentariums. Le rôle que tiennent les deux instruments baroques varie considérablement au fil des sections: ils peuvent être totalement absents ou apparaître comme solistes; ils peuvent instaurer, avec les instruments modernes, un dialogue apaisé ou au contraire assez musclé; ils peuvent aussi totalement faire corps avec eux... Ces différentes formes d'interaction n'étaient pas préalablement définies mais découlent du travail d'écriture sur les différents matériaux. Elles dessinent ainsi les contours d'un scénario musical que le compositeur affine grâce à un travail de montage extrêmement précis, qu'il soit à l'échelle de l'œuvre ou à l'échelle des différentes sections qui la composent: «[...] j’ai coupé, taillé, remonté et assemblé. Je travaille beaucoup avec la technique de montage, en fait. [...] Avec l'utilisation des outils informatiques, et notamment le programme de saisie musicale Finale, j'ai pris l'habitude de manipuler, déplacer, retravailler, agencer les différentes sections ou objets musicaux déjà composés. Je me rends compte maintenant que j'ai vraiment besoin de passer par cette étape de travail car je ne cherche pas forcément une continuité. Je travaille avec des fragments et des unités composées qui, par le jeu d'agencement dans la pièce, se rejoignent petit à petit. »

FIGURE 2 David Hudry (à gauche) lors d'une séance de répétition dans un studio de l'Ensemblehaus Freiburg en juillet 2016 en vue de l'enregistrement de Störungen ${ }^{12}$. Avec l'aimable autorisation de l'ensemble recherche. Photographie: (c) Beate Rieker.

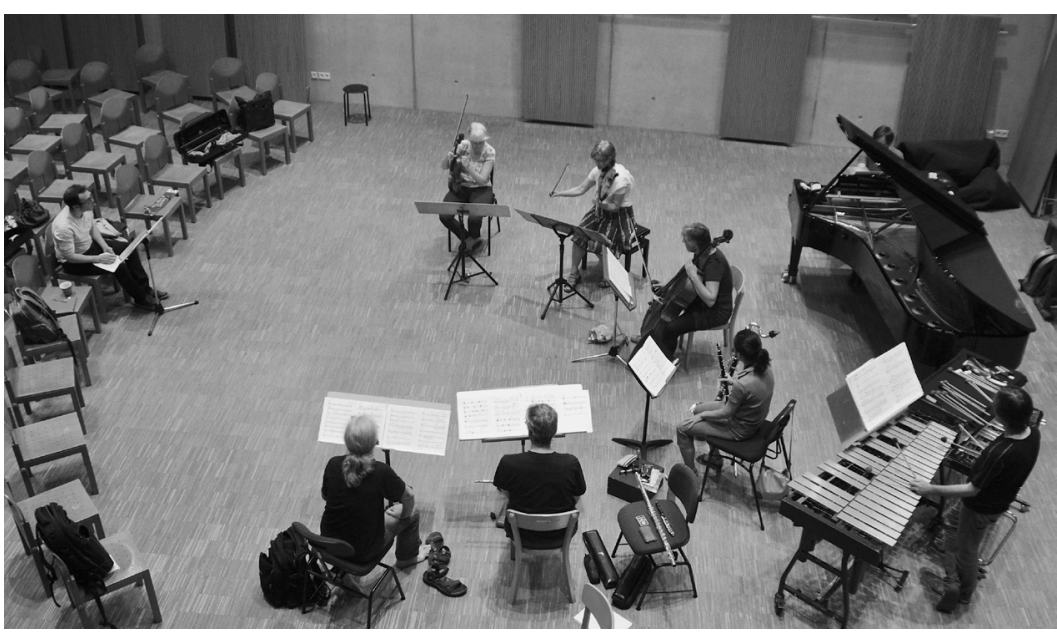


FIGURE 3 Aperçu synthétique de l'évolution du discours musical au fil des différentes sections de Störungen ${ }^{13}$.

\begin{tabular}{|c|c|c|}
\hline Section & Repères & Résumé \\
\hline A & $\begin{array}{l}\text { Partition: m. 01-66 } \\
\text { Audio: 00'00-03'14 }\end{array}$ & $\begin{array}{l}\text { Juxtaposition de trois types de séquences au cours desquelles le } \\
\text { traverso n'apparaît jamais: } \\
\text { - Séquences pulsées, dynamiques et denses jouées par les six } \\
\text { instruments modernes et recouvrant un large ambitus avec } \\
\text { l'utilisation de la flûte piccolo et de la clarinette basse. } \\
\text { - Séquences plus calmes et aérées où l'alto baroque fait figure de } \\
\text { soliste. } \\
\text { - Séquences statiques écrites en parfaite homorythmie et issues de } \\
\text { la section C; l'alto et le violoncelle forment un double qui fusionne } \\
\text { parfaitement avec le piano, le vibraphone et le violon. }\end{array}$ \\
\hline B & $\begin{array}{l}\text { Partition: m. 67-137 } \\
\text { Audio: 03'14-04'54 }\end{array}$ & $\begin{array}{l}\text { Le traverso et la flûte moderne se mêlent et s'entremêlent tout } \\
\text { au long de cette section pour donner naissance à une seule } \\
\text { ligne mélodique virtuose avec des accents incisifs produits par } \\
\text { la technique d'over-blowing }{ }^{14} \text {. Soit le violon ajoute, par bribes, de } \\
\text { courts motifs mélodiques, esquissant les débuts d'un contrepoint; } \\
\text { soit il se greffe à l'alto baroque, au violoncelle et au vibraphone, } \\
\text { pour introduire un nouvel élément d'essence ternaire qui contraste } \\
\text { avec l'hydre mélodique façonnée par les deux flûtes. }\end{array}$ \\
\hline C & $\begin{array}{l}\text { Partition: m. 138-170 } \\
\text { Audio: 04'54-06'12 }\end{array}$ & $\begin{array}{l}\text { Tous les instruments à vent sont mis au repos dans cette section } \\
\text { dont le caractère statique et homorythmique tranche avec ce qui } \\
\text { précédait. L'alto baroque joue à l'octave du violoncelle (octave } \\
\left.\text { qui n'en est pas une en raison des diapasons différents }{ }^{15}\right) \text {. Les } \\
\text { harmoniques du violon, les sons graves du piano dont les cordes } \\
\text { ont été étouffées et les sons aigus du glockenspiel viennent colorer } \\
\text { la ligne mélodique. }\end{array}$ \\
\hline D & $\begin{array}{l}\text { Partition: m. 171-244 } \\
\text { Audio: 06'12-08'45 }\end{array}$ & $\begin{array}{l}\text { Le discours est ici très articulé et dynamique grâce à la trame } \\
\text { pulsée jouée par la clarinette, la flûte et le piano. Le traverso se } \\
\text { détache tel un soliste de cette trame avant que celle-ci ne l'absorbe } \\
\text { progressivement. L'alto baroque ne fait en revanche pas figure de } \\
\text { soliste; il se mélange aux autres cordes à quelques reprises pour } \\
\text { jouer des éléments préalablement entendus comme les motifs } \\
\text { d'essence ternaire de la section B ou des extraits de la section C. }\end{array}$ \\
\hline E & $\begin{array}{l}\text { Partition: m. } 245^{-} \\
330 \\
\text { Audio: } 08^{\prime} 45-13^{\prime} 25\end{array}$ & $\begin{array}{l}\text { Cette section est sans aucun doute la plus concertante de l'œuvre. } \\
\text { L'alto baroque, après un long solo que l'on peut assimiler à une } \\
\text { cadence, cherche à s'affirmer face aux autres instruments qui } \\
\text { reprennent différents matériaux de l'œuvre. Le traverso n'apparaît } \\
\text { que très rarement en revanche, et jamais en tant que soliste; sa } \\
\text { présence est liée à la reprise de certains passages rythmés de la } \\
\text { section D. }\end{array}$ \\
\hline $\mathbf{F}$ & $\begin{array}{l}\text { Partition: } \mathrm{m} \cdot 331- \\
364 \\
\text { Audio: } 13^{\prime} 25-15^{\prime} 40\end{array}$ & $\begin{array}{l}\text { Les instruments sont sur un pied d'égalité dans cette section où } \\
\text { plane l'ombre de Vortex Temporum (1994-1996) de Gérard Grisey. } \\
\text { Basée sur une série d'accords formulés sous forme d'arpèges et } \\
\text { suivant des balancements qui se décalent les uns par rapport } \\
\text { aux autres, cette section semble illustrer la réconciliation entre } \\
\text { les instrumentariums baroque et moderne d'une part, et entre les } \\
\text { différents matériaux de l'œuvre qui sont alors entendus de manière } \\
\text { superposée et non plus juxtaposée. }\end{array}$ \\
\hline G & $\begin{array}{l}\text { Partition: m. } 365-403 \\
\text { Audio: } 15 \text { '40-17'08 }\end{array}$ & $\begin{array}{l}\text { Dans l'esprit d'une coda, le compositeur continue de faire } \\
\text { réapparaître subrepticement les principaux matériaux de son } \\
\text { œuvre... dernières réminiscences avant que le rideau ne se ferme. }\end{array}$ \\
\hline
\end{tabular}

13. Pour le complément web accompagnant cet article: https:// www.revuecircuit.ca/web/ (consulté le 3 mai 2018), nous avons sélectionné six extraits de Störungen accompagnés de la partition et enrichis des commentaires du compositeur issus de notre entretien avec lui.

14. L'over-blowing - littéralement "sur-souffle" - est une technique de jeu consistant à forcer le souffle pour obtenir un des harmoniques de la note jouée et enrichir le son avec le souffle de l'interprète.

15. Puisque les instruments baroques sont accordés suivant un diapason à $415 \mathrm{~Hz}$, ils sonnent un demi-ton en dessous des instruments modernes accordés à $440 \mathrm{~Hz}$. L'unisson entre les deux instrumentariums sonne donc comme un intervalle de seconde mineure et l'octave comme une octave diminuée ou augmentée suivant que les instruments baroques sont situés respectivement à l'octave supérieure ou inférieure des instruments modernes. 
L'enregistrement de l'œuvre qui figure sur le disque monographique Durchgang a été réalisé le 2 juillet 2016 dans un des studios de l'Ensemblehaus Freiburg (Figure 2). Le tableau prédédent propose un résumé synthétique de la trame narrative de Störungen (Figure 3). Le sonagramme et la forme d'onde obtenus à partir de l'enregistrement rendent compte du travail de montage opéré par le compositeur et du contraste saisissant entre les différentes sections (Figure 4).

FIGURE 4 Forme d'onde et sonagramme (FFT sur 20000 échantillons, plage temporelle en abscisse : 17'06”, plage fréquentielle en ordonnée: $6000 \mathrm{~Hz}$ ) obtenus avec le logiciel Audiosculpt à partir de l'enregistrement de Störungen (col legno WWE 1 CD 40418, 2017).

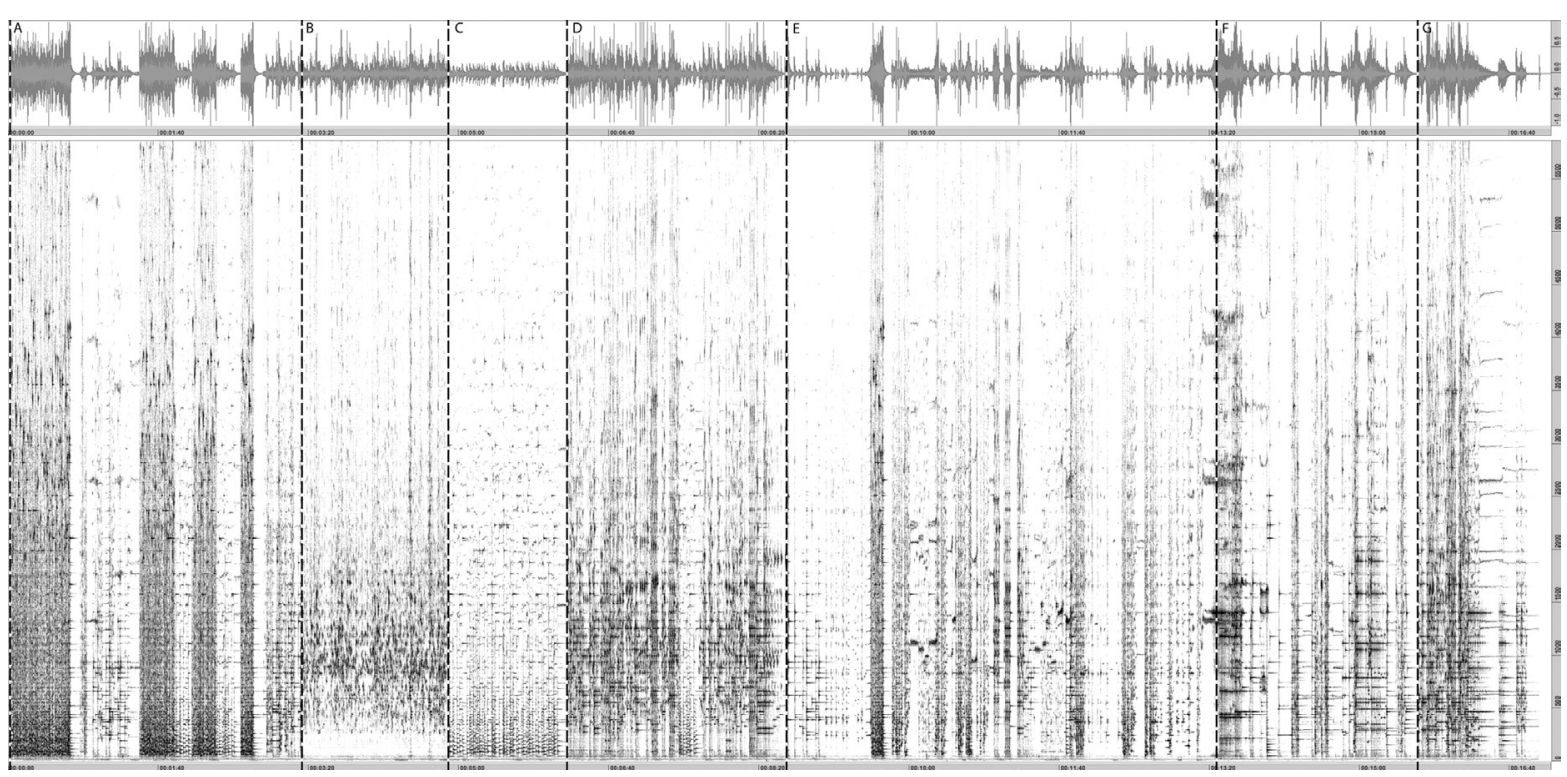

\section{Unification, dédoublement et vagabondage : les différents rôles des deux instruments baroques}

Le rôle que tiennent les deux instruments baroques au sein du discours musical varie sans cesse au fil de l'œuvre. Lalto baroque, bien plus que le traverso, fait souvent figure de soliste, comme lors de son entrée en scène (Section A, m. 17-27) (Figure 5, à gauche), ou bien dans la cadence (Section

16. Verwandlung I (2006), pour alto solo, a été créée en mai 2007 par Christophe Desjardins dans le cadre du festival "Les Musiques", organisé par le GMEM-CNCM-Marseille.
E, m. 250-258) dont le matériau initial dérive d'une œuvre antérieure du compositeur écrite pour alto solo ${ }^{16}$. "Pour une raison que je ne pourrai pas formuler clairement, j'ai toujours associé l'alto à un personnage solitaire, à l'idée d'âme solitaire. Je parle souvent dans ma musique de ma relation à Schubert. J'aime énormément Schubert, sa musique et le thème du Wanderer qui parcourt son œuvre. Dans cette pièce, l'alto a le rôle de personnage solitaire, de Wanderer, 
FIGURE 5 Deux extraits de Störungen dans lesquels l'alto baroque (m. 18-19 à gauche) et le traverso (m. 191-194 à droite) font figure d'instruments solistes.

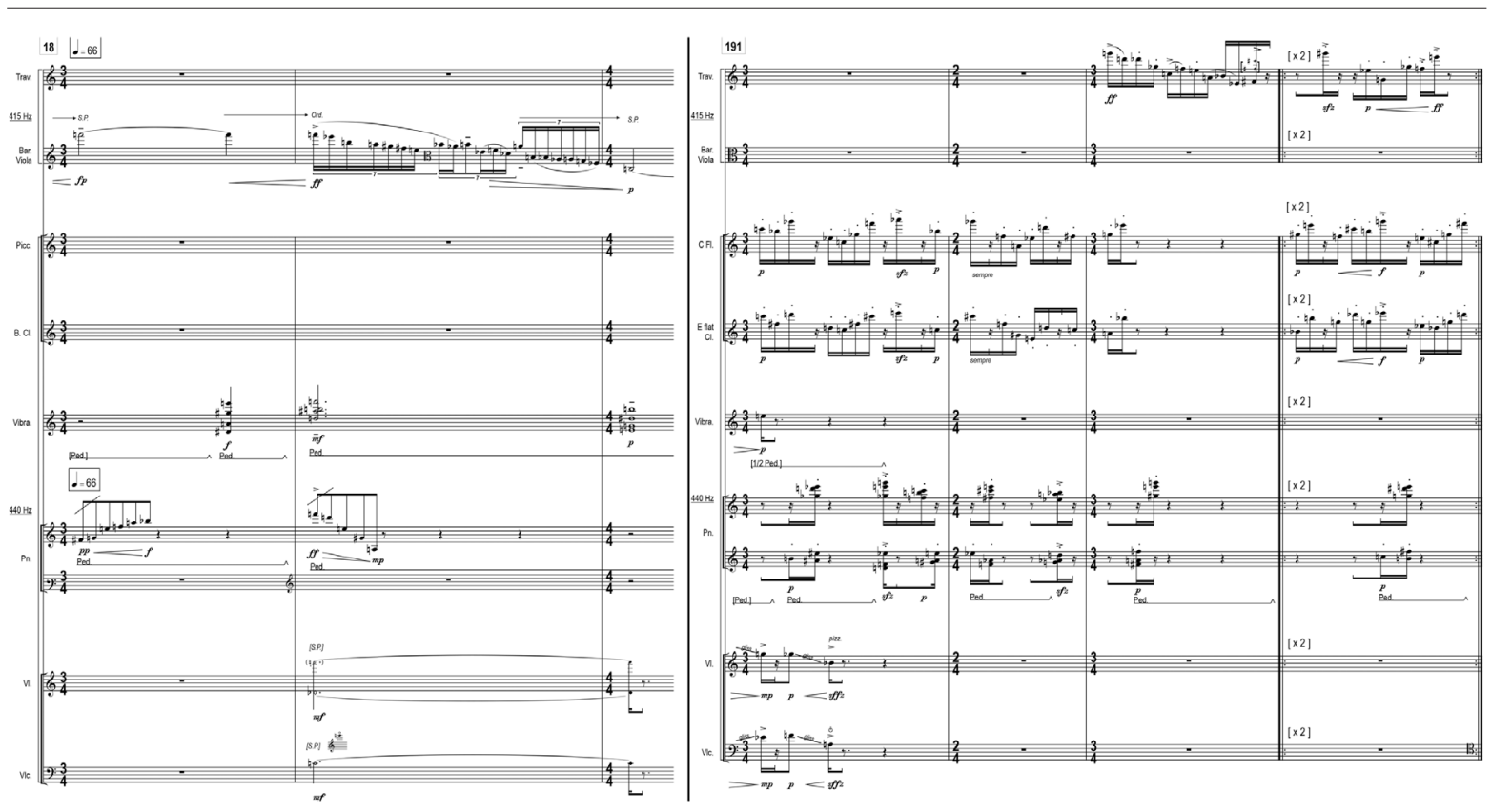

qui se promène et se questionne. Il a un rôle presque romantique. » Si David Hudry n'écrit aucune cadence pour le traverso, celui-ci apparaît néanmoins à plusieurs reprises comme un instrument soliste, notamment dans la section D où il s'émancipe des autres instruments avant de se mêler de plus en plus à eux, telle une ombre qui se détacherait ou se rapprocherait de son sujet (Figure 5, à droite).

Lidée de dédoublement et de duplication, qui intéresse beaucoup David Hudry depuis quelques années ${ }^{17}$, se traduit dans Störungen de diverses façons pour explorer de nouvelles formes de dysfonctionnement: « Le double représente soit un personnage avec de multiples visages ou personnalités, soit au contraire, un autre moi dans un autre espace. » Au dédoublement des diapasons s'ajoute ainsi un dédoublement instrumental, le traverso étant très souvent couplé à la flûte traversière et l'alto baroque au violoncelle. À de multiples reprises, le compositeur écrit à l'unisson ou à l'octave pour ces paires d'instruments mais, en raison des différents diapasons, ces intervalles supposés purs sont en réalité dénaturés, grossissant ainsi le trait des lignes mélodiques ou des harmonies. "Il y avait cette idée du double, d'une part parce qu'il y avait le lien avec le concerto, et d'autre part, parce que dans ma façon de composer, j'imagine souvent un instrument soliste comme étant 
FIGURE 6 Début de la section C de Störungen (m. 138-141) accompagné de son sonagramme (FFT sur 20000 échantillons, plage temporelle en abscisse: 10", plage fréquentielle en ordonnée: $1150 \mathrm{~Hz}$ ) obtenu avec le logiciel Audiosculpt à partir de l'enregistrement de Störungen (col legno

18. En raison de son accordage à $415 \mathrm{~Hz}$, l'alto baroque enrichit considérablement le spectre sonore en ajoutant un nouveau système de fréquences à celui produit par les instruments modernes qui jouent le plus souvent à l'octave les uns des autres. Nous avons indiqué sur le sonagramme - au niveau des sons longs - les composantes harmoniques correspondant aux notes jouées par le violoncelle (en blanc) et par l'alto baroque (en noir). À la mesure 140, exceptionnellement, l'altiste ne produit pas la note escomptée dont nous avons indiqué les composantes théoriques (en gris) mais semble jouer à l'octave réelle du violoncelle.

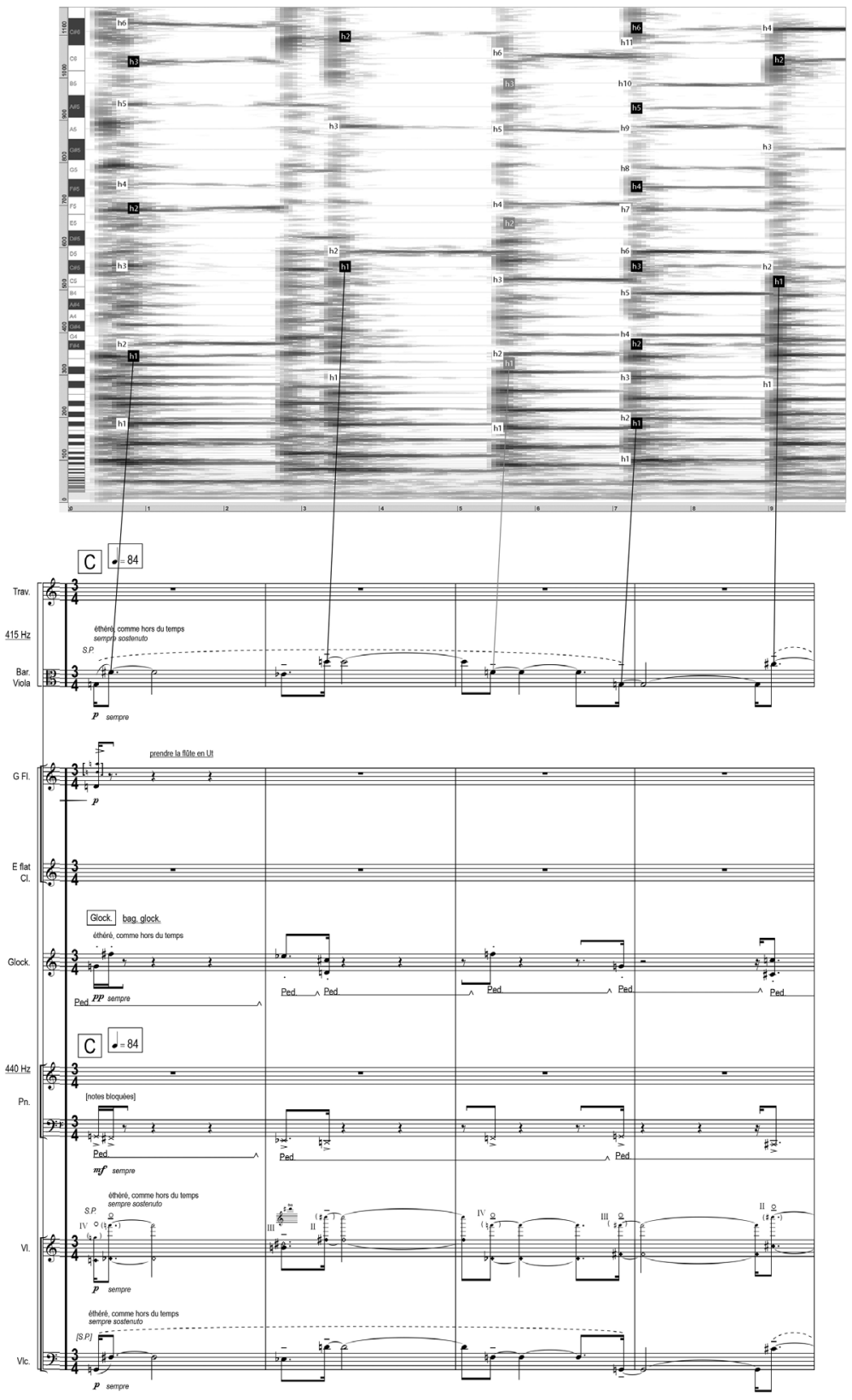


accompagné ou suivi par une ombre, une trace, quelque chose qui lui donne une épaisseur, une ampleur particulière. Du coup, le jeu sur les diapasons couplé au travail sur des unissons ou des octaves permettait une épaisseur à des lignes mélodiques auxquelles j'étais particulièrement attaché.»

Dans la section C par exemple, le violoncelle (sul ponticello) déploie une lente mélodie orchestrée par le violon (en harmonique), le piano (avec les cordes étouffées), le glockenspiel et l'alto baroque (sul ponticello), tous jouant en parfaite homorythmie. Le compositeur privilégie les rapports d'octave en cherchant à couvrir un large ambitus. Lalto baroque joue systématiquement à l'octave du violoncelle pour former un «seul et même personnage dédoublé». Mais cette octave, qui est diminuée en raison des différents diapasons, n’est pas ici perçue comme un intervalle mais produit plutôt un effet de distorsion qui vient colorer et épaissir la ligne mélodique pour mieux la voiler d'un halo de mystère (Figure 6).

FIGURE 7 Imbrication des lignes mélodiques jouées par les deux flûtes dans la section B de Störungen (m. 68-75).

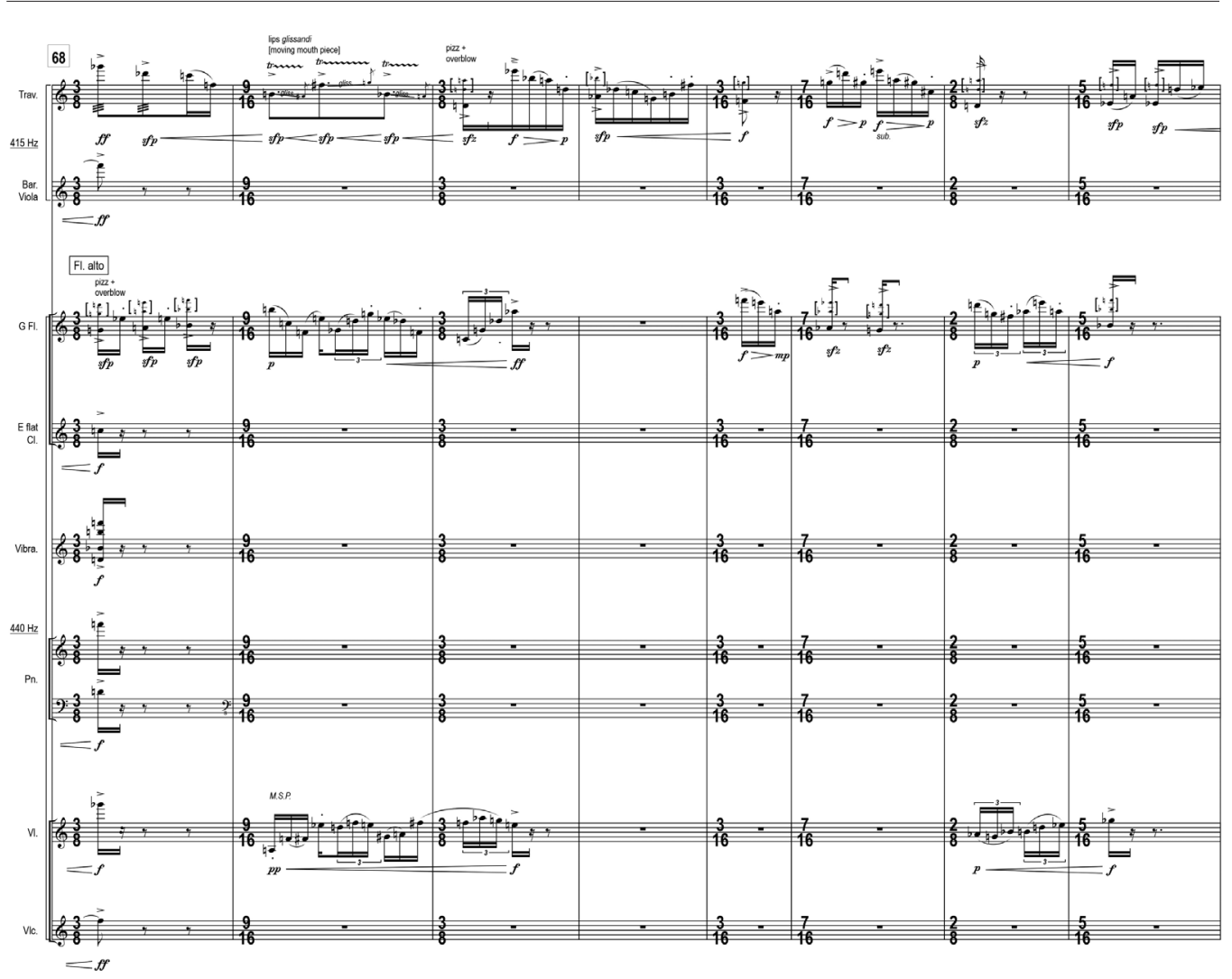


Le compositeur explore aussi son idée de dédoublement d'une tout autre manière en entremêlant plusieurs instruments pour donner naissance à «un seul personnage ayant plusieurs facettes». Lorsqu' il apparaît pour la première fois dans l'œuvre (section B, m. 67), le traverso est ainsi totalement enchevêtré à la flûte moderne. Il devient impossible de discerner les deux instruments individuellement, ceux-ci concourant à la formation d'une unique image auditive au contour instable... une sorte d'hydre mélodique. Tout au long de cette section, les deux instrumentistes se relaient ou se superposent frénétiquement, usant de l'over-blowing pour enrichir le son et s'approcher du timbre du shakuhachi ou des flûtes des Andes. "J'ai demandé à la flûte, et surtout au traverso pour qui cette technique n'est pas du tout habituelle, de jouer avec beaucoup d'air et beaucoup de transitoire d'attaque afin de produire la couleur spécifique désirée dans ce passage. » De temps à autre, le violon vient s'insérer, donnant l'impression de vouloir arbitrer en vain le combat que se livrent les deux flûtistes (Figure 7).

\section{Enjeux et défis relatifs à cette nouvelle forme de mixité instrumentale}

Si réunir instruments anciens et modernes permet d'enrichir la palette des timbres et de créer de nouveaux alliages, cela n'est pas sans poser quelques problèmes pour les compositeurs qui doivent non seulement intégrer la différence des diapasons mais aussi tenir compte des caractéristiques acoustiques des instruments baroques, ainsi que des pratiques interprétatives qui leur sont propres.

Dans Störungen, la différence de diapason d'un demi-ton tempéré entre instruments baroques et modernes était imposée. Mais si d'autres projets de la sorte se présentaient à lui, David Hudry opterait pour une différence de un ou deux tiers de ton afin de pouvoir «travailler plus sur les phénomènes de battements, de dédoublement progressif». Quelle que soit cette différence, le compositeur doit constamment faire l'effort de transposer les parties des instruments baroques pour se représenter les relations harmoniques avec les instruments modernes. Et pour mieux appréhender l'effet perceptif des intervalles issus de cet alliage, il lui est arrivé de réaliser quelques simulations par ordinateur en recourant à des échantillons de sons réels, notamment pour la construction de la section C. "Ie jouais avec un échantillonneur des fragments mélodiques au diapason et puis un autre que j'essayais de transposer pour avoir une idée de comment ça allait fonctionner. Je faisais cela avec un séquenceur dans lequel je rentrais des notes avec de vrais échantillons d'instruments et puis je les écoutais. [...] D’abord, j'ai transposé la même ligne, après j'ai fait des expériences en changeant la hauteur des échantillons pour écouter. La superposition de la ligne mélodique originale à ses transpositions légèrement 
modifiées m'a permis d'apprécier les effets produits sur la ligne mélodique principale. [...] Évidemment, il n'y a pas que la question de l'écart entre les notes; il y a également celle du timbre, qui peut difficilement être simulée sans les instruments réels à disposition. Les différences de timbres dépendent aussi du registre de l'instrument. Je me souviens, par exemple, que le son de l'alto baroque devenait beaucoup plus tendu dans l'aigu que sur un alto classique, justement à cause des cordes en boyau.»

L'équilibre instrumental entre les voix est une dimension importante que le compositeur avoue ne pas avoir bien anticipée. S'il était évidemment conscient que les instruments baroques étaient moins puissants que les instruments modernes, ce n'est qu'au moment des répétitions, à travers l'expérience de l'écoute, que les problèmes d'équilibre se sont pleinement révélés. "Je pense avec le recul que je n'avais pas assez anticipé la capacité de projection de la flûte baroque par rapport à la flute moderne et j'ai fait ce constat au moment où les musiciens ont commencé à répéter ensemble. À vrai dire, au début de la composition du projet, j'avais pensé régler le problème en éloignant les instruments baroques et les plaçant vraiment au-devant de la scène, ce qui répondait à l'idée initiale de "Concertare". Mais, sans chef pour diriger la pièce, cela rendait la mise en place compliquée.»

David Hudry n’a pu travailler, en amont de la composition de l'œuvre, qu'avec le flûtiste baroque Karl Kaiser qui lui avait tout d'abord fourni par écrit quelques explications (ambitus, série de doigtés...) avant qu'ils ne se rencontrent, à Paris, en janvier 2010. Le compositeur a alors pu mieux intégrer les spécificités de cet instrument. «On a fait une session de travail et d'enregistrement à partir d'esquisses que je lui avais proposées. J'ai pu mieux apprécier ses possibilités, et les contraintes ou difficultés liées à la facture de l'instrument. J'ai pris le parti de corriger certaines choses qui auraient été gênantes pour la musique, et j'en ai conservé d'autres malgré les difficultés qu'elles posaient, car elles me semblaient nécessaires pour exprimer le caractère que je voulais donner à cette lutte entre le traverso et le reste de l'ensemble.»

Par sa complexité, l'écriture de David Hudry ne ménage pas toujours les musiciens. Dans l'esprit de Brian Ferneyhough, il lui arrive de recourir à une écriture solfégique qui vise, d'une certaine manière, à les mettre en danger. «Je crois qu'à cette époque-là, c'était ma façon à moi aussi de chercher cette forme de tension, de moments où on est à la lisière avec la rupture.» Cette virtuosité prend une nouvelle dimension dès lors qu'elle s'applique à des instruments baroques. Le traverso, par exemple, est un instrument diatonique si bien qu'il n'est pas aisé, sur le plan digital, de réaliser des enchaînements chromatiques rapides comme ceux que privilégie le compositeur dans Störungen. Aller ainsi 
19. Dans les années 1990 sous l'impulsion de Michaël Levinas, L'Itinéraire a organisé une série de concerts intitulée "Répliques", mélangeant musiques anciennes sur instruments d'époque et créations contemporaines. Plus récemment, l'ensemble s'est associé au département de musique ancienne du CRR de Paris et a organisé, le 20 mars 2017, un concert intitulé "Le présent et ses doubles" au cours duquel a été créée Doppio Trio de Noriko Baba pour 3 instruments anciens et 3 instruments modernes. L'Ensemble intercontemporain et Les Arts Florissants - tous deux résidents à la Cité de la musique - Philharmonie de Paris - ont de leur côté proposé, en 2015, des rencontres avec le public intitulées "Qui se ressemble s'assemble". à l'encontre des spécificités instrumentales lui permettait d'explorer une fois de plus l'idée de dysfonctionnement. «Je crois que d'une certaine manière, j'ai été attiré par une forme de virtuosité, de défi instrumental dans lequel je voulais pousser le musicien baroque au-delà de ses habitudes traditionnelles de jeu. Je crois même que dans cette pièce, j’en ai fait un peu une obsession, ce qui rend la pièce pas forcément facile à jouer. Pour traduire cette idée de dysfonctionnement et de perturbation, il m'a fallu inventer des situations musicales qui permettaient de ressentir ces états d'instabilité et de les opposer aux moments de repos. C'est la façon que j'ai trouvée à l'époque pour exprimer ces effets de ruptures."

Certaines techniques de jeu, telles que l'over-blowing, ne sont pas employées dans le répertoire ancien et sont plus difficiles à réaliser d'ailleurs au traverso qu'à la flûte moderne. Le musicien baroque doit alors s'affranchir des canons de son instrument. "J'ai dû lui demander d'exagérer toutes les articulations et tous les modes d'émission du son pour parvenir à donner le caractère que je cherchais. Karl voulait absolument faire un son très propre alors que j’avais besoin au contraire d'énergie. Je lui ai même demandé de ne pas hésiter à salir le son en rajoutant plus de souffle. C'est finalement lorsqu'il s'est laissé aller sans chercher à contrôler la propreté du son que j'ai eu les attaques et l'énergie nécessaires pour exprimer le caractère de lutte. Ça marchait beaucoup mieux!»

\section{Épilogue : perspectives pour l'émancipation d'un répertoire mêlant instruments anciens et modernes}

Les liens entre musiques anciennes et musiques d'aujourd'hui se sont naturellement renforcés ces dernières années, notamment par le biais d'interprètes qui s'investissent avec autant de passion et d'aisance dans ces répertoires séparés de plusieurs siècles. Il n’est plus rare de voir des ensembles spécialisés partager la même scène pour jouer leur répertoire respectif au cours d'un même concert et parfois s'unir pour assurer ensemble la création d'une œuvre. L'Ensemble Academy Freiburg, créé en 2004 sous l'impulsion du Freiburger Barockorchester et de l'ensemble recherche favorise ainsi les échanges entre instrumentistes baroques et modernes et concoure sûrement d'une certaine façon à un décloisonnement des publics. Bien qu'il n'ait jamais été reconduit faute de subventions publiques (Féron, 2018b), le concours de composition «Concertare», organisé en 2010, a contribué au développement d'un répertoire encore trop ténu mais plein de promesses, où se mêlent instruments anciens et modernes accordés selon des diapasons différents. Des initiatives du même genre commencent d'ailleurs à voir le jour dans d'autres pays, notamment en France autour de formations instrumentales comme par exemple L'Itinéraire, l'Ensemble intercontemporain et Les Arts Florissants ${ }^{19}$. 
Si ce type de mixité instrumentale peut paraître problématique à certains égards, il ouvre un vaste champ de possibles d'un point de vue compositionnel comme en témoigne Störungen de David Hudry. Mais pour parfaire de tels projets, souligne le compositeur, ceux-ci devraient s'inscrire dans le cadre de résidences artistiques au cours desquelles des séances de travail communes pourraient être organisées avec les instrumentistes baroques et modernes. «Lidéal serait d'avoir des temps de rencontre où l'on peut préparer des fragments, les faire jouer, les écouter, revenir dessus et retravailler en fonction de la réalité acoustique. [...] Sur des projets de cette nature, et encore plus lorsque l'on expérimente ce genre de formation pour la première fois, il est important de se faire une idée sonore concrète des contraintes imposées par des instruments spécifiques»... un vœu pieux mais néanmoins raisonné si l'on veut que ce répertoire puisse pleinement s'émanciper et fédérer autour de lui différents musiciens mais aussi différents publics.

\section{REMERCIEMENTS}

Je souhaiterais exprimer toute ma gratitude à David Hudry pour sa disponibilité et sa confiance, à Tanja Ratzke (directrice générale de l'ensemble recherche) pour les précieuses informations qu'elle m’a communiquées au sujet de l'Ensemble Academy Freiburg et à Christian Faurens (SCRIME) pour la confection des vidéos du supplément web. Enfin, je souhaiterais remercier chaleureusement la Ernst von Siemens Music Foundation, l'étiquette col legno et l'ensemble recherche pour m'avoir autorisé à reproduire des extraits sonores de Störungen dans ce supplément.

\section{B IBLIOGRAPHIE}

FÉRon, François-Xavier (2018a), entretien privé avec David Hudry, Enghien-les-Bains, 4 janvier.

FÉron, François-Xavier (2018b), entretien privé par courriels avec Tanja Ratzke, directrice générale de l'ensemble recherche (janvier-février).

Merlin, Arnaud (2017), «Le portrait contemporain - David Hudry, de la dramaturgie à la pulsation", émission radiophonique sur France Musique, 20 décembre 2017. https:// www.francemusique.fr/emissions/le-portrait-contemporain/david-hudry-compositeur-38794 (consulté le 3 avril 2018).

Rigaudière, Pierre (2017), «David Hudry's Musical World-Visions », livret du CD Durchgang (col legno WWE 1 CD 40418), p. 42-55.

\section{DISCOGRAPHIE ET PARTITION}

Hudry, David (2011), Störungen for mixed ensemble consisting of baroque and modern instruments, disponible sur la bibliothèque en ligne de musique contemporaine Babelscores.

Hudry, David (2017), Durchgang, Ensemble moderne, ensemble recherche, Orchestre philharmonique de Radio France, Ensemble intercontemporain, dir. Duncan Ward, Pascal Rophé et Bastien Stil, col legno WWE 1 CD 40418. 